\title{
Miniature Arcs for Synthesis of Carbon Nanotubes in Microgravity
}

\author{
J.M. Alford ${ }^{*}$ and G.R. Mason ${ }^{\zeta}$ \\ TDA Research, Inc. \\ 12345 W. $52^{\text {nd }}$ Ave. \\ Wheat Ridge, CO 80228 \\ D.A. Feikema \\ NASA Glenn Research Center \\ Cleveland, $\mathrm{OH} 44135$
}

\begin{abstract}
Although many methods are available for producing single-walled carbon nanotubes (SWNTs), the conventional carbon arc process remains the most popular due to its simplicity and large production rate. In the carbon arc, SWNTs are catalytically synthesized by rapidly evaporating a graphite anode impregnated with Ni/Y metal catalyst from which the nanotubes grow in an inert atmosphere. However, high temperatures inside the carbon arc generate strong buoyancy driven convection, and it is hypothesized that the non-uniform environment created by this flow has a large effect on the growth and morphology of the SWNTs. To study the effect of buoyancy on the arc process, a miniature carbon arc apparatus was developed to synthesize SWNTs in a microgravity environment substantially free from these strong convective flows. The reactor was operated for either 2.2- or 5-seconds during free-fall in the drop towers at the NASA Glenn Research Center. Two apparatus designs differing mainly in their production rate and power capacity were investigated. The first consisted of a miniaturized carbon arc employing a $1 \mathrm{~mm}$ diameter graphite anode and powered by a $0.54 \mathrm{~F}$ capacitor bank charged to $65 \mathrm{~V}$. The second, larger apparatus employed a 4 $\mathrm{mm}$ diameter anode and was powered by a portable battery pack capable of providing in excess of 300 amps at 30 volts to the arc for the duration of a 5-second drop. Initial results indicated that transient heating is a very large effect in the short-duration drop tower carbon arcs, and thermal equilibrium of the arc plasma, buffer gas, and apparatus was not attained during the short microgravity periods. In addition, removal of the buoyant convection by the microgravity now allowed clear observation of large jets of evaporated carbon vapor streaming from the anode and mixing with the inert buffer gas. The initial mixing of these jets with the cold buffer gas combined with the thermal transient made it difficult to establish a uniform high temperature environment around the arc in the 2.1- to 5-second microgravity time interval, and even with a very high-powered arc, the arc region was cooler than in continuously operated arcs. Despite these difficulties, the miniature arc produced SWNTs in microgravity. However, given the large thermal transient to overcome, no dramatic difference in sample yield or composition was noted between normal gravity and 2.2- and 5-second long microgravity runs.
\end{abstract}

\section{INTRODUCTION}

Because of their potential as new electronic and mechanical materials, there is currently a large interest in the synthesis and characterization of carbon nanotubes. Multi-walled carbon nanotubes approximately $2 \mathrm{~nm}$ in diameter, (ten times smaller than conventional carbon fibers), were first observed in the carbon arc by Iijima in 1991. Addition of a transition metal catalyst to the carbon arc allowed production of single-walled carbon nanotubes $^{2,3}$ (SWNTs) with diameters typically between 1.2-1.5 nm. Unlike conventional carbon fibers, the SWNT structure is atomically perfect, thus producing defect free fibers that have many enhanced electronic, thermal, and mechanical properties. ${ }^{4-6}$ With inherently low weight and a predicted Young's modulus of $\sim 1$ terapascal, (five times that of steel), SWNTs are expected to have many uses in composite materials. For example, high strength PBO (poly p-phenylene benzobisoxazole) fibers similar to Zylon demonstrate a $50 \%$ increase in tensile strength when doped with $10 \%$ SWNTs, $^{7}$ and recent processing breakthroughs now allow neat SWNTs to be spun into pure,

* Senior Scientist, Non AIAA Member

$\zeta$ Mechanical and Electrical Technician, Non AIAA Member

* Aerospace Research Engineer, Senior Member AIAA

Copyright (C) 2006 by TDA Research Inc. and NASA GRC. Published by the American Institute of Aeronautics and Astronautics, Inc. with permission. 
continuous fibers. ${ }^{8}$ SWNTs have a thermal conductivity equal to diamond ${ }^{9}$ suggesting their use for heat transfer. For electronics, specific tubes have conducting electronic structures and can be envisioned as molecular "quantum" wires exhibiting low resistance ballistic conduction, ${ }^{10}$ while others are semiconducting and can be used to fabricate "single molecule" field effect transistors ${ }^{11}$ and logic circuits. ${ }^{12}$ Non-volatile memory chips ${ }^{13}$ made from SWNTs are close to commercialization. Fluorescent lights employing nanotube field emission are now commercially produced,${ }^{14}$ and flat panel field emission displays are currently in development. Other potential uses for SWNTs include: tips for atomic force microscopy, chemical sensors, molecular containers, and catalyst supports.

There are currently several methods for producing single-walled carbon nanotubes, including the carbon arc discharge, ${ }^{15}$ laser vaporization of graphite, ${ }^{16}$ chemical vapor decomposition ${ }^{17}$ (CVD), high-pressure disproportionation of $\mathrm{CO}$ (HiPco), ${ }_{18}$ and flame synthesis. ${ }^{19}$ Although detailed formation mechanisms for each of these methods remain unknown, all rely on catalytic growth of the nanotube from either pure carbon or hydrocarbon vapor in the presence of $\mathrm{Co} / \mathrm{Ni}$ or other transition metal catalysts. For high volume commercial applications, such as composite materials, large amounts of material at economical prices are required. In this regard, the carbon arc, chemical vapor deposition, and disproportionation of CO methods currently appear to be the most commercially viable. The laser vaporization technique generates very high quality nanotubes, but the production rate is low and the cost of the laser is high. The flame method is intriguing due to its similarity to commercial carbon black production, but currently it remains a relatively low yield experimental process.

While the CVD and HiPco processes appear to be promising methods for future nanotube production, scale up for both remains in the developmental stage. Nanotube CVD, which relies upon supported catalyst particles, has proven very effective for growing tubes in specifically defined arrays or patterns for electronic devices and field emission applications. However, the use of CVD to produce bulk quantities of unsupported, free standing SWNTs remains in development. Likewise, the HiPco process also remains under development, and a recent review article ${ }^{18}$ reports production rates of $10 \mathrm{~g}$ per day. Because of its simplicity and bulk production capability, the original carbon arc discharge method remains a popular and competitive process, especially for scientific and $R \& D$ applications. Although the arc discharge technique is a batch process, recent experiments with large, 3-phase plasma arcs demonstrate the capability of continuously processing kilograms of carbon materials per hour. ${ }^{20}$ The prospect of using such large arc discharges for the continuous production of carbon nanotubes provides substantial motivation for us to increase our understanding of the arc discharge synthesis of carbon nanotubes. To this end, we have designed and tested a miniature carbon arc discharge reactor for use in a microgravity environment.

\section{A. II. THE NEED FOR MICROGRAVITY}

Ideally, nanotube growth should be studied in a plug flow reactor with uniform flow and a well-controlled temperature gradient that allows the temperature and reaction time to be precisely controlled. Unfortunately, the high temperature needed to vaporize carbon, which is higher than all other structural materials, precludes the use of conventional chemical reactors. Instead, carbon vapor must be sublimed directly from the carbon electrodes of the arc discharge, generating plasma temperatures in excess of 5,000-10,000 ${ }^{\circ} \mathrm{C}$. However, at these temperatures, the density of the plasma and carbon vapor may be an order of magnitude less than the surrounding gas, producing large convective flows. Computer modeling confirms that gas velocities between 1 and $2 \mathrm{~m} / \mathrm{sec}$ are rapidly established around the arc. ${ }^{21}$ Compared to the dimensions of the arc discharge, typically less than $1 \mathrm{~cm}$, a convective flow of several $\mathrm{m} / \mathrm{sec}$ rapidly carries the nanotube reactants out of the high temperature region in $100 \mathrm{msec}$ or less. The growth time and temperature for SWNTs is not known precisely, but it could be as long as several seconds under favorable conditions. ${ }^{22}$ In this case, the convective flow dramatically shortens the residence time available for tube growth. In addition, the flow is highly irregular, causing the time temperature history for trajectories initiating from different portions of the arc to be drastically different, therefore making it difficult to determine and maintain the optimum reaction conditions.

Comparison of results for fullerene and nanotube production using arc discharges shows substantial variations in the yield of fullerenes and nanotubes collected from various parts of the arc chamber, consistent with large convection. This has prompted several experimental investigations on the influence of gravitational convection on the arc discharge. Under normal gravity, Aoyama and Mieno have found that orientation of the carbon arc has a large influence on the production of $\mathrm{C}_{60}$ fullerenes; a large drop in the $\mathrm{C}_{60}$ yield was observed when the anode (positive electrode) of the DC arc was placed at the top of a vertical arrangement. ${ }^{23}$ In a DC carbon arc, sublimation of the carbon occurs almost exclusively at the anode due to electron bombardment, and the drop in yield was 
attributed to the convective flow, which cooled and re-deposited the sublimed carbon back onto the anode. Similarly, Marin et al. have reported enhanced production of SWNTs by placing the anode at the bottom of the vertical arrangement to promote convective assisted deposition of the nanotubes on the cathode. ${ }^{24}$ The use of magnetic fields to direct the plasma flux using the $\overrightarrow{\mathbf{J}} \times \overrightarrow{\mathbf{B}}$ force has also been shown to affect the production rate and yield of fullerenes and SWNTs. ${ }^{23,25}$ When the plasma jet was magnetically directed along the direction of natural convection, the production rate and yield of SWNTs dramatically increased, while directing the jet against the convective flow caused the production rate and yield to drop. It was conjectured that the downward jet interacts with the upward convective flow disrupting the nanotube reaction zone. Convection clearly has a large, usually detrimental effect on fullerene and nanotube synthesis in the carbon arc, and we hypothesize that convection rapidly carries the reactants away from the hot zone and facilitates mixing with the cold background gas, reducing the time that the reactants are at the temperature needed for nanotube and fullerene growth.

Much more uniform reaction conditions dominated mainly by molecular diffusion can be obtained by completely removing thermal convection using zero gravity conditions. The plasma should then form a larger, symmetric sphere of hot gas around the electrodes, producing a much more uniform reaction zone. Heat from the arc should be retained in this zone, producing a substantially longer reaction time. The first experiments to attempt convection free arc discharges for fullerene and nanotube production were conducted by Mieno using an arc reactor with an oscillating weak gravity environment produced by a vertical swing tower. ${ }^{26-28}$ The reported oscillation period was $2.3 \mathrm{sec}$ with a gravity free time of $1.1 \mathrm{sec}$ per oscillation. The gravity free condition was reportedly better than $0.05 \mathrm{G}$ and was followed by a deceleration that reached $2 \mathrm{G}$. The arc was run only during the low gravity periods for a total of 20-30 minutes. The results were highly encouraging and showed that the metallofullerene and nanotube production rate and yield was substantially enhanced by reduced gravity. SWNTs grown under reduced gravity also showed a change in diameter distributions to larger sizes and an increase in purity. ${ }^{28}$ Convection in this type of apparatus is substantially reduced but not entirely eliminated. After the initial reduced gravity period, the arc is stopped, but the electrodes and surrounding plasma, which are still at high temperatures, will initiate convective flow during the enhanced gravity deceleration period. Once initiated, momentum will continue to circulate these currents inside the arc chamber during the next period. An additional limitation of this experiment is the short arc discharge time. Since the arc discharge is only on for 1.1 seconds, the size of the spherical reaction zone is restricted. Because the molecular weight of the nanotubes is very high, diffusion through the reaction zone will be slow, and it is possible that nanotube growth could be limited by the short arc time. Given the promising results of the swing tower, more sophisticated experiments are warranted.

Longer periods of higher quality reduced gravity can be produced by several methods including drop towers, aircraft flying parabolic trajectories, and sounding rockets. Given the large size, weight, and power requirements of the arc discharge apparatus, drop towers and zero-G aircraft represent the best choices for future experimentation. Reports of experiments to develop carbon arc discharges for zero-G aircraft, ${ }^{29,30}$ and the evolution of arc discharge temperatures generated under reduced gravity in an aircraft have been reported. ${ }^{29}$ Aircraft have the advantage of producing up to 20 seconds of reduced gravity in multiple cycles, but access to the aircraft is limited and expensive. Reduced gravity on the aircraft is typically $10^{-3} \mathrm{G}$, and structural vibrations of the aircraft and apparatus can cause G-jitter, a problem noted to adversely affect the electrodes of the arc discharge flown on a zero-G aircraft. ${ }^{30}$ Drop towers provide true microgravity that is actually better than that on the space shuttle and space station and are generally more accessible and economical than aircraft. Microgravity for our arc experiments was provided by 2.2second $^{31}$ and 5- second ${ }^{32}$ drop towers located at the NASA Glenn Research Laboratory in Cleveland, OH. The 2.2second drop tower afforded a reduced gravity of $\sim 100 \mu \mathrm{G}$ using an aerodynamic drag shield during free fall for 24.1 $\mathrm{m}$. A reduced gravity of $\sim 1 \mu \mathrm{G}$ for 5.18 seconds was provided by a $131.7 \mathrm{~m}$ free fall under millitorr vacuum conditions at the NASA Glenn Zero Gravity Facility.

\section{THE DROP TOWER MICROGRAVITY ARC APPARATUS}

Use of an arc discharge in a drop tower imposes tight dimensional, weight, and power requirements. To operate on both the 2.2-second and 5-second drop towers, the arc apparatus must fit a frame measuring $96 \mathrm{~cm}$ long by $40 \mathrm{~cm}$ deep by $84 \mathrm{~cm}$ high. Weight is restricted to $160 \mathrm{~kg}$, and all power for the arc and associated instrumentation must be totally self-contained. In the 5- second drop tower, the apparatus and instrumentation must be compatible with the external vacuum used to remove wind resistance. In addition, the apparatus must survive a deceleration of up to 65 
G's at the end of the run. The short duration of the drop experiment means we must design an apparatus with a high rate of production so that sufficient nanotube material is generated for meaningful analysis.

In addition to miniaturization, the conventional, ground-based reactor had to be substantially re-designed to meet these requirements. In the following sections, we detail the mini arc electrodes, the specially doped graphite, and the circuits and power supply needed to run the apparatus. A schematic of the reactor used in the 5- second drop tower is shown in Figure 1.

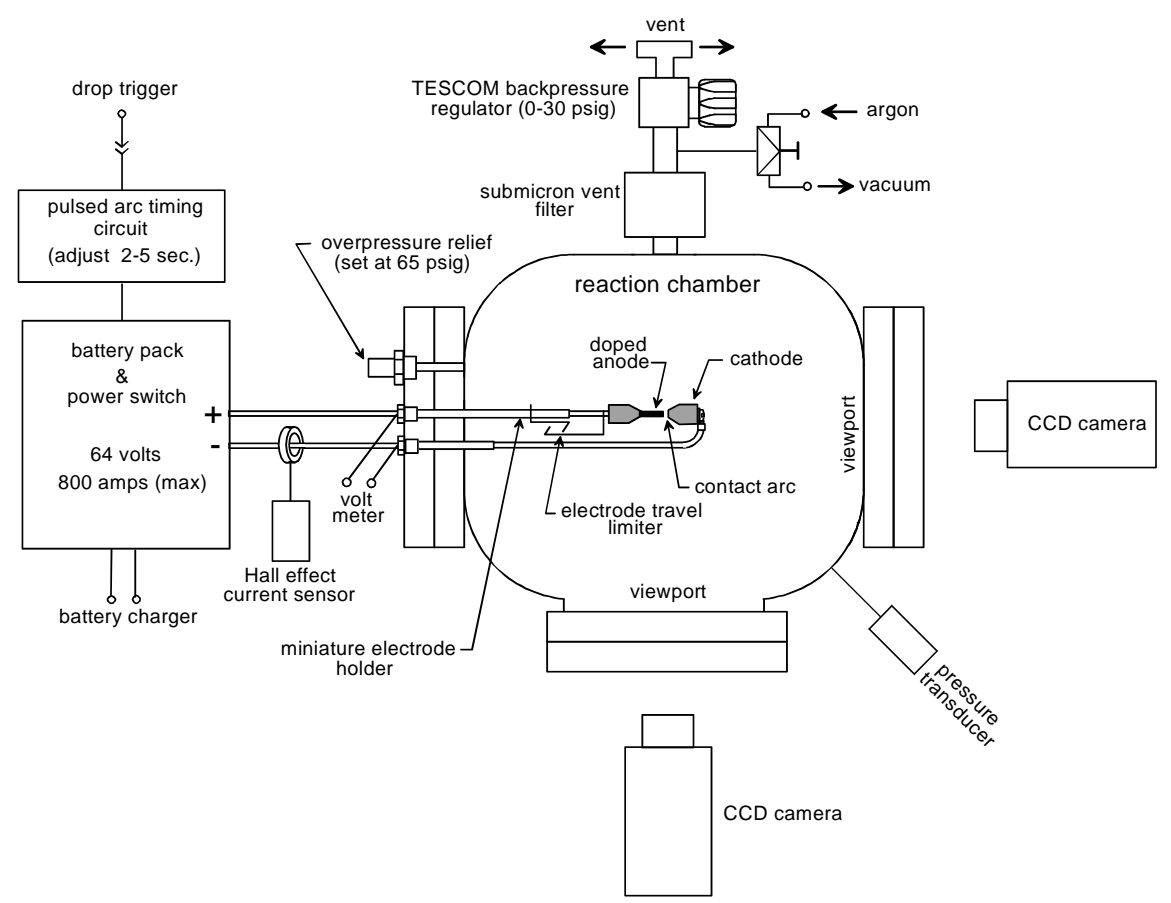

Figure 1 Schematic of the miniature microgravity carbon arc reactor used in the Zero Gravity Facility (ZGF) 5.18 second drop tower. Chamber dimensions $\sim 20 \mathrm{~cm}$ in diameter.

Conventional Arc Electrodes

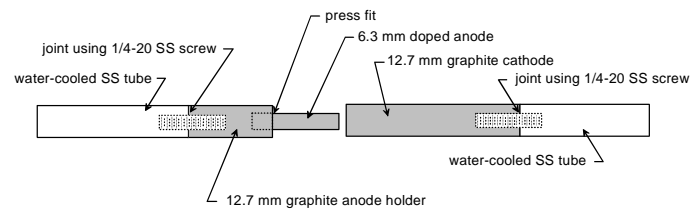

Miniature Arc Electrodes

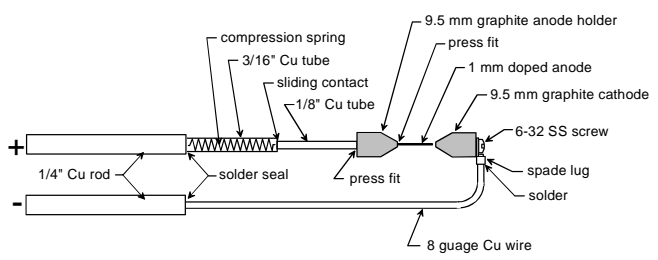

Figure 2 Upper: The arc electrodes used in full size, conventional arc apparatus for nanotube production. Lower: The miniature arc electrodes used in the drop tower experiment hardware. 


\section{A. Miniature arc concepts}

To determine the operating parameters such as current, power, and production rate required for the microgravity arc, SWNTs were first produced under normal gravity according to literature methods ${ }^{15}$ using a conventional arc apparatus with electrodes shown in Figure 2. A catalytic metal doped anode was fashioned by drilling a $2 \mathrm{~mm}$ diameter hole through a $6.3 \mathrm{~mm}$ diameter x $50 \mathrm{~mm}$ deep graphite $\operatorname{rod}^{33}$ and packing the hole with a mixture of $\mathrm{Y}_{2} \mathrm{O}_{3}$ and $\mathrm{NiO}$ powder wetted with polyfurfural alcohol, producing an electrode containing about $4 \%$ by atom $\mathrm{Ni}$ and $1 \%$ Y. The rod was baked at $2000{ }^{\circ} \mathrm{C}$ under a dynamic vacuum of 20 mtorr for ten minutes to carbonize the PFA binder and reduce the $\mathrm{Ni}$ and $\mathrm{Y}$ oxide to elemental metal and metal carbide. The doped rod was then press fit $10 \mathrm{~mm}$ deep into a larger $12.7 \mathrm{~mm}$ diameter by $50 \mathrm{~mm}$ long graphite holder and was arced against a $12.7 \mathrm{~mm}$ diameter by 100 $\mathrm{mm}$ long pure graphite cathode using DC current from a high performance TIG welding power supply. The arc discharge was performed in 25 liter bell jar chamber filled with 600 torr of Ar held at constant pressure by a control valve. During arcing, a small gap of $\sim 3 \mathrm{~mm}$ was maintained between the electrodes, and the current was maintained at 125 amps. The measured voltage across the arc averaged $25 \mathrm{~V}$, and the entire $40 \mathrm{~mm}$ doped length was evaporated in two minutes. The effect of convection was immediately apparent as web-like strands of carbon fibers could be seen blowing and circulating in the chamber. Analysis of the soot by high-resolution transmission electron microscopy HR-TEM showed a large yield of SWNTs, consistent with Journet et al. ${ }^{15}$

The conventional arc continuously dissipated $\sim 3$ kilowatts of power, giving a current and power density at the anode of about $4.4 \mathrm{~A} / \mathrm{mm}^{2}$ and 110 Watts $/ \mathrm{mm}^{2}$, respectively. Graphite was evaporated at an average rate of about $700 \mathrm{mg} /$ minute or $\sim 12 \mathrm{mg} / \mathrm{sec}$. Sublimation of carbon produces an equilibrium mixture of $\mathrm{C}_{1}-\mathrm{C}_{5}$ clusters with the most abundant species being $\mathrm{C}_{3}, \mathrm{C}_{5}$, and $\mathrm{C}_{2}$. Equilibrium calculations at 600 torr using thermochemical data for $\mathrm{C}_{1}$ $\mathrm{C}_{5}$ indicate that sublimation occurs near $3660{ }^{\circ} \mathrm{C}$ and requires $280 \mathrm{~kJ} /$ mole of enthalpy. ${ }^{34}$ At steady state, a simplified thermal analysis neglecting convection indicates that only 300 watts are required to vaporize the graphite, while the remaining 2.7 kilowatts goes into producing blackbody radiation, heating the Ar gas, and thermal conduction through the electrodes. Heat loss through the electrodes is estimated to be $435 \mathrm{~W}$ for the current geometry assuming the anode tip is at the sublimation point of graphite while the cathode is cooler at $3000{ }^{\circ} \mathrm{C}$. Heating the Ar gas in the chamber to $600{ }^{\circ} \mathrm{C}$ over the two-minute period requires only $50 \mathrm{~W}$. The remaining majority of power, $2.2 \mathrm{~kW}$, is dissipated as radiation, which is consistent with the size of the electrodes and plasma and noting that ideal blackbody emission is $1.3 \mathrm{~kW} / \mathrm{cm}^{2}$ at the sublimation point of carbon and $4.4 \mathrm{~kW} / \mathrm{cm}^{2}$ for 5000 ${ }^{\circ} \mathrm{C}$ plasma. With only 2.2 -seconds of production time available in the short drop tower, the thermal transient time required to heat the electrodes at the start of the arc also must be taken into account. Considering only the heat capacity of the electrodes, $\sim 7 \mathrm{~kJ}$ is needed to heat the anode to its steady state temperature distribution, while $49 \mathrm{~kJ}$ is needed to heat the larger cathode. If we designed a drop tower experiment to operate with the same power input as the steady-state laboratory unit, the $3 \mathrm{~kJ} / \mathrm{sec}$ of input power would not be sufficient to establish thermal equilibrium of the electrodes in 2.2 , or even 5 -seconds, and the region around the microgravity arc would never reach the operating temperature of the full gravity reactor.

\section{B. Miniaturized arc electrodes}

Considerable re-design was required to produce an apparatus suitable for the drop tower. The time required to heat the electrodes of the conventional arc apparatus is too long, and although large in size, the conventional arc does not make efficient use of the available power to vaporize the graphite. An average evaporation rate of 12 $\mathrm{mg} / \mathrm{sec}$ will produce only small amounts of sample in 2 seconds, and this rate most likely occurs after thermal equilibrium is established. To allow quicker heating of the electrodes, their thermal mass must be substantially reduced. To make better use of the power for evaporation of the graphite, the plasma discharge volume must also be reduced to decrease the amount of radiation. Two approaches were investigated to overcome these limitations, and both relied on substantial miniaturization of the entire arc electrode assembly as illustrated in Figure 2.

\section{B.1 2.2-second arc}

In the first design, intended for initial testing in the 2.2-second drop tower, the anode was shrunk to $1 \mathrm{~mm}$ in diameter (retaining the active length) reducing its cross sectional area and mass by a factor of 40 . The volume of radiating plasma, which depends on the cross sectional area of the anode, was also reduced accordingly. The radiating surface area, which scales linearly, was reduced by a factor of six. The $12.7 \mathrm{~mm}$ diameter anode holder 
and cathode were reduced to $9.5 \mathrm{~mm}$ in diameter, shortened to $15 \mathrm{~mm}$ long, and angled as shown to reduce the thermal mass by an additional factor of ten. The thermal conductivity of the electrode assembly was lessened by approximately four times by using 0.125 " copper tube to supply current to the anode and 8-gauge (0.128") solid copper wire to connect the cathode. Electrical resistance was dominated by the $1 \mathrm{~mm}$ graphite rod, which had a resistance of $\sim 0.75 \Omega$ compared to several $\mathrm{m} \Omega$ for the remainder of the electrode.

Radiation losses were further reduced and evaporation efficiency was increased by changing the discharge from a large gap to a contact arc design. Most large arc discharge machines operate with an arc gap of 3-5 mm, which is adjusted manually by the operator or automatically using a feedback loop based on arc current. The extended gap produces a large plasma volume and a correspondingly large radiation power loss. Early experiments on fullerene production showed that a small electric arc could produce large yields of fullerenes ${ }^{35}$ using a contact arc. Instead of a large gap and plasma, the contact arc utilizes the large resistance at the junction of the anode and cathode to generate a small, confined arc in the tiny gaps between the mating surfaces. Radiation from the contact arc is reduced and falls mainly on the ends of the electrodes, making evaporation of the graphite much more efficient. The contact arc has the additional advantage of being self-starting upon initiation of the current and does not require mechanical regulation of a specific gap distance, which would be very difficult given the short 2 to 5- second arc duration. Early contact arc designs employed a vertical orientation using gravity as the driving force to maintain the contact arc. Our electrode utilized a sliding seal with an internal custom wound spring to supply an average contact force of $10 \mathrm{~g}$-force over $40 \mathrm{~mm}$ of travel. Electrical contact was provided by a $6 \mathrm{~cm}$ long sliding contact formed from a $1 / 8$ " precision $\mathrm{Cu}$ tube sliding inside a $3 / 16$ " precision $\mathrm{Cu}$ tube. The sliding contact electrical resistance was less than $10 \mathrm{~m} \Omega$ and did not appear to degrade during repeated runs. When mounted on a $1 / 2$ " Plexiglas end flange using bored out 1/4" pipe fittings, the electrode assembly survived repeated $65 \mathrm{G}$ decelerations with no damage.

Energy for the small arc was supplied by a $0.544 \mathrm{~F} 100 \mathrm{~V}$ capacitor bank that stored a maximum of $2.72 \mathrm{~kJ}$ of energy. In addition to supplying a compact, very low internal resistance energy source compatible with the drop tower size and weight requirements, capacitors have the additional advantage in that the exponential decay of current provides a simple method to partially compensate for the transient delay required for heating the electrodes.

Experiments showed that consistently evaporating $15 \mathrm{mg}$ of the carbon anode in 2.1 seconds (slightly shorter than the drop time) required $64 \mathrm{~V}$ and $1.1 \mathrm{~kJ}$ of energy in the capacitor. The capacitor did not completely discharge, and about $1 \mathrm{~kJ}$ was actually consumed. The total resistance of the arc circuit including the electrodes, power switch, cabling, and internal resistance of the capacitor was approximately $0.95 \Omega$. Assuming constant resistance, $210 \mathrm{~J}$ was used to heat the $0.20 \Omega$ external circuit resistance, leaving $790 \mathrm{~J}$ entering the electrode. Of this energy, vaporizing the carbon required a minimum of $350 \mathrm{~J}$ for the enthalpy of sublimation. Even with substantially reduced thermal mass, it is estimated that 250-350 J could be needed for heating the electrodes. Therefore, supplying energy into the electrode at the estimated average rate of $375 \mathrm{~W}$ could require nearly one second of the available microgravity time for transient heating. However, using the ideal exponential discharge of the capacitor from $64 \mathrm{~V}$ with a RC time constant of $540 \mathrm{msec}$, produces an initial current of $64 \mathrm{~A}$ and allows the first $350 \mathrm{~J}$ for transient heating to be delivered in only 0.26 second. It should be noted, though, that the actual discharge current and power delivery rate is more complicated because the resistance as well as the voltage is time varying. Initial heating causes a quick rise in resistance of the graphite, while vaporization of the graphite shortens the anode decreasing the resistance. Measurements showed the current starting at $65 \mathrm{~A}$ and ending after 2.1 seconds at about $15 \mathrm{~A}$, which is a much higher ending current than predicted from the RC constant, and it shows a substantial increase in the time constant after the initial inrush of current. The available energy in this approach was limited to about $1 \mathrm{~kJ}$ because the inrush current at higher voltages produced such rapid heating that thermal shock literally exploded the electrodes into several large pieces.

\section{B.2 5-second arc}

The use of a simple capacitor bank for power allowed a series of quick 2.2-second survey experiments to be conducted with the mini-electrodes. However, the nanotube yield was very small and did not increase in microgravity. Examination of video from 2.1 second duration arcs in microgravity showed that the graphite evaporated well for the first 1-1.5 seconds then decreased significantly as the capacitor discharged and the input power dropped. The drop in evaporation rate and small total energy raised concerns that the plasma sphere and surrounding reaction zone was cooling too rapidly to grow nanotubes. Coupled with the small $15 \mathrm{mg}$ samples, this prompted development of a much more powerful miniature arc for use in the 5-second drop tower. An ambitious 
goal of producing a $500 \mathrm{mg}$ sample in 5- seconds suggested that the power would need to be increased by at least 15 times from $350 \mathrm{~W}$ to $5250 \mathrm{~W}$ and held steady for the entire 5 -second discharge. Given the restricted size and weight requirements, development of a totally self-contained power supply of this capacity presented a considerable challenge.

A power supply was developed (described in detail below) that could easily generate an arc discharge with a current of $300 \mathrm{~A}$ and voltage of $35 \mathrm{~V}$ and deliver a total power of $10.5 \mathrm{~kW}$ to the electrode for the entire 5-second duration. Use of such high currents required several minor modifications of the electrode. The cathode and anode holders were increased from $9.5 \mathrm{~mm}$ to $12.7 \mathrm{~mm}$ in diameter while the doped anode was increased from $1 \mathrm{~mm}$ to 4.7 $\mathrm{mm}$ in diameter. All other dimensions remained unchanged. The $1 / 8$ " precision copper tube holding the anode assembly was changed to solid rod to increase its current carrying capacity, and a new spring was wound to supply $120 \mathrm{~g}$-force for the contact arc. Initial testing showed that the sliding contact exhibited some surface erosion at these currents, so a bypass conductor made from 8 gauge flexible copper braid was added to connect the $1 / 4$ " solid rod to the $1 / 8$ " rod. Electrical resistance of the assembly was reduced to approximately $0.1 \Omega$. Increasing the mass of the anode again increased the energy needed for transient heating, but with $10 \mathrm{~kW}$ of power and 5 -seconds of microgravity, a heating time of $0.5-1$ second was not so critical, and no pre-emphasis of the current pulse was attempted. Testing showed that the high current power supply and miniature electrodes could easily evaporate 100$200 \mathrm{mg} / \mathrm{sec}$ of carbon, over ten times the rate of the much larger conventional arc.

\section{Catalytically doped graphite}

Production of carbon nanotubes is a catalytic reaction in which high energy carbon clusters or amorphous graphite dissolve in a transition metal, $\mathrm{Fe}, \mathrm{Co}$, or $\mathrm{Ni}$, catalyst, and the carbon is re-crystallized as a more stable nanotube structure. The size and type of nanotube, single-walled or multi-walled, is believed to depend on the catalyst particle size and temperature. The optimum catalyst composition appears to depend highly on the experimental conditions. A breakthrough in SWNT production yield for arc discharges was obtained by Journet $e t$ $a l .{ }^{15}$ who added $\mathrm{Y}$ to the $\mathrm{Ni}$ to increase the yield. The $\mathrm{Y}$, or other lanthanide elements, is presumed to form metallofullerene structures at high temperatures, which then condense with the Ni clusters at lower temperatures, thus increasing the yield of nanotubes. ${ }^{36}$ The optimum ratio of catalysts for typical arc discharges was found to be 4.2 atom $\% \mathrm{Ni}$ and 1 atom $\% \mathrm{Y}$, which was added to the graphite by simply drilling holes in the graphite electrode anode and packing it with mixtures of catalyst as described previously. It is assumed, but not established that this mixture evaporates evenly into the plasma with the surrounding graphite.

The miniature arc requires doped graphite anodes that are too small to make by the drilling and packing method. Ideally, the catalyst would be homogeneously incorporated into the graphite during its production, but custom graphite production is not feasible for most laboratories. Our solution was to synthesize doped graphite from high purity, porous graphite (POCO PS-1 grade with $\sim 40 \%$ porosity) using an incipient wetness impregnation technique originally developed for metallofullerene production. ${ }^{37}$ The densest possible impregnation solution was prepared by

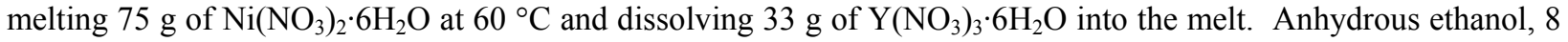
$\mathrm{ml}$, was then added to allow the solution to wet the graphite and penetrate the pores. PS-1 graphite rods 4.7 and 6.3 $\mathrm{mm}$ in diameter were evacuated to $100 \mathrm{mtorr}$ in a heated container, and nitrate solution was added through a rubber septum until the rods were totally immersed. The rods were then soaked in the melted solution for several hours. After cooling, the rods were removed and air-dried for 12 hours. To remove the ethanol and further solidify the impregnated salt, the rods were dried in a vacuum furnace at $45{ }^{\circ} \mathrm{C}$ for $36 \mathrm{hrs}$. To remove the hydrated water without re-melting the salt, a slow temperature ramp from 45 to $220^{\circ} \mathrm{C}$ over 60 hours was applied, and the rods were then held at $220^{\circ} \mathrm{C}$ for an additional 6 hours. A final temperature ramp from 220 to $1000{ }^{\circ} \mathrm{C}$ over 15 hours with an additional hour at $1000{ }^{\circ} \mathrm{C}$ was used to convert the nitrates to oxide. To verify the uniformity and composition, random pieces were cut from several rods, digested in acid, and analyzed for Y and Ni using ICP spectroscopy. The results confirmed a uniformly doped composition containing 1.05 atom $\% \mathrm{Ni}$ and 0.27 atom \% Y. This is about four times less than that recommended by Journet et al., ${ }^{15}$ but was the maximum that could be obtained given the graphite pore volume and solution density. Because of the lower concentration, the $6.3 \mathrm{~mm}$ rods were tested in the conventional arc apparatus where they produced a better yield of SWNT than the drill and pack approach. This is likely due to increased efficiency from having a much more uniform distribution of catalyst in the graphite, suggesting that the standard assumption of uniform evaporation in the pack and drill approach could be 
incorrect. Smaller $1 \mathrm{~mm}$ diameter by $50 \mathrm{~mm}$ long rods were then cut from the larger rods while the $4.7 \mathrm{~mm}$ rods were cut to a $50 \mathrm{~mm}$ length and used as is.

\section{Arc discharge power supply}

All power for drop tower experiments must be totally self-contained on the drop rig, limiting energy sources to batteries and capacitors. The power supply described below received several design iterations as the power capacity was increased, but the timing and trigger circuits remained unchanged. For 2.2-second drops requiring several kilojoules of energy, a capacitor bank made from eight $68,000 \mu \mathrm{F} 100 \mathrm{~V}$ electrolytic capacitors in parallel proved more than adequate, but represents about the limit of this approach. Batteries remain the only other viable power source, but arc discharges require very large currents and power, necessitating a very low internal battery resistance. Conventional lead acid storage batteries utilizing large electrode plates can supply the power and current requirements, but are too heavy and lack the mechanical strength to survive the $65 \mathrm{G}$ deceleration. The vents on such batteries are also not compatible with the vacuum in the 5- second drop tower. However, lead acid battery resistance can be substantially reduced by employing optimized thin electrode geometries constructed from high purity tin and lead. The Enersys G13EP, for example, is a $13 \mathrm{~A}-\mathrm{hr} 12 \mathrm{~V}$ lead acid battery with a short circuit current of $1400 \mathrm{~A}$ ( $8.5 \mathrm{~m} \Omega$ internal resistance) while having dimensions of just $17.5 \mathrm{~cm}$ long by $8.3 \mathrm{~cm}$ wide by $13 \mathrm{~cm}$ high and $4.9 \mathrm{~kg}$. This battery also has a vent rated for vacuum, is nonspillable, and survived repeated drop tower decelerations. A $64 \mathrm{~V}$ compact battery pack consisting of five G13EP batteries connected by copper bars and weighing only $30 \mathrm{~kg}$ was constructed for drop tower use. Using the power switch described below, it could supply a constant current in excess of $800 \mathrm{~A}$ at $30 \mathrm{~V}$ into a short circuit load for more than 5-seconds (limited by the power supply, connections, and wire resistance).

Triggering and timing of the arc power to coincide with release of the drop rig is controlled by the pulse circuit shown in Figure 3. The discharge sequence is initiated by pullout of a drop trigger fashioned from a banana plug. A 30 millisecond delay pulse is then generated by $1 / 2$ of a 74LS221 TTL Schmitt triggered multivibrator chip to allow vibrations from the release mechanism to damp before the actual power pulse (adjustable from 1-5 seconds) is generated by the second half of the 74LS221. The power timing pulse is then sent through an MCT2 optical isolator and a 4049 CMOS inverting buffer to drive the gates of ten power MOSFETs in parallel. For simplicity, no attempt was made to actively control the current, and the FETs were driven as switches into their full on state. Experiments needing different arc currents were performed by changing the voltage of the capacitor bank or by changing the internal resistance of the battery pack by adjusting the charge level in the batteries. The use of optical isolation and a floating battery power supply for the 4049 buffer allows the MOSFETs to be driven in a bootstrap arrangement where the gate voltage floats on the changing source or load voltage, thus maintaining the gate source voltage necessary for the FETs to remain in the full on position. With this arrangement, the batteries are connected to the arc electrodes only when the transistors are turned on, thereby increasing safety. The use of optical isolation also decreases electrical noise from the arc and prevents re-triggering of the pulse timer circuit.

Efficiently delivering power into the arc discharge requires a switch with an extremely low internal resistance due to the small resistance of the arc $(0.1 \Omega)$. The absolute current limit comes from the batteries, which are rated to safely withstand a direct short circuit current of $1400 \mathrm{~A}$. With $64 \mathrm{~V}$ and a $42.5 \mathrm{~m} \Omega$ internal battery pack resistance, the theoretical maximum current and power for a $0.1 \Omega$ arc discharge is about $600 \mathrm{~A}$ and $40 \mathrm{~kW}$. Producing a more modest $10 \mathrm{~kW}$ arc discharge requires $316 \mathrm{~A}$ and a power switch with a resistance of $60 \mathrm{~m} \Omega$ and power capacity of 6 $\mathrm{kW}$. Because the large amount of energy, $\sim 1 \mathrm{MJ}$, stored in the battery pack could potentially destroy the apparatus if inadvertently discharged, the switch was required to be substantially over-rated and to safely handle a direct short circuit of its outputs. Therefore, a switch composed of a large number of parallel transistors was selected to divide up the current load and add redundancy. In addition, all devices were run well under their rated capacity.

The switch for the capacitor powered arc utilized ten $200 \mathrm{~V} 30$ A IRF250 power FETs ( $85 \mathrm{~m} \Omega$ of resistance) in parallel, and each device was current limited by a $2.5 \Omega$ drain resistor providing a total resistance of $0.26 \Omega$. At 100 $\mathrm{V}$, the IRF250s operate completely within their safe operating area even with the output shorted. The much higherpowered battery driven circuit, shown in Figure 3, employed ten parallel FB180SA10 modules rated for $100 \mathrm{~V}$ and $180 \mathrm{amps}$ with a resistance of $6.5 \mathrm{~m} \Omega$. Current limiting was insured by a $0.25 \Omega$ drain resistance made from four $1.0 \Omega 50 \mathrm{~W}$ power resistors in parallel for each module. The total resistance was about $38 \mathrm{~m} \Omega$ versus $42.5 \mathrm{~m} \Omega$ for the battery pack internal resistance. The combined resistance, which also includes the wiring, limits the short circuit 
current to about 80 A per module and ensures a large safety margin. When shorted, the power through the drain resistors is approximately ten times their rated continuous capacity, but no problems were noted since it was a single pulse with only a 5 -second duration. However, over $120 \mathrm{~kJ}$ must be dissipated, and all components were securely mounted with thermal grease to a large aluminum heat sink. This is especially important for use in the drop tower vacuum chamber, as natural convection can no longer cool the components. To further minimize resistive loses, current from the transistor bank was delivered to the arc electrodes using eight-gauge wire.

The battery-powered circuit also contained voltage and current monitoring instrumentation as shown in Figure 3. The arc discharge voltage drop was monitored across the electrodes where they entered the vacuum chamber using an Analog Devices AD628 precision difference amplifier designed for accurate differential sensing across inputs floating at up to $+/-120 \mathrm{~V}$. The AD628 provides stable gains less than 1 and was configured with a gain of 0.05 to provide a $0-5$ volt signal for analog to digital conversion. The current was monitored with a F.W. Bell CLN-1000 (0-1000 A) closed-loop Hall effect sensor, which functioned as a current source producing $0.2 \mathrm{~mA} / \mathrm{A}$ of current passing through the sensor loop. This was converted to a $0-5$ voltage ( $0.01 \mathrm{~V} / \mathrm{A})$ using a second AD628 and

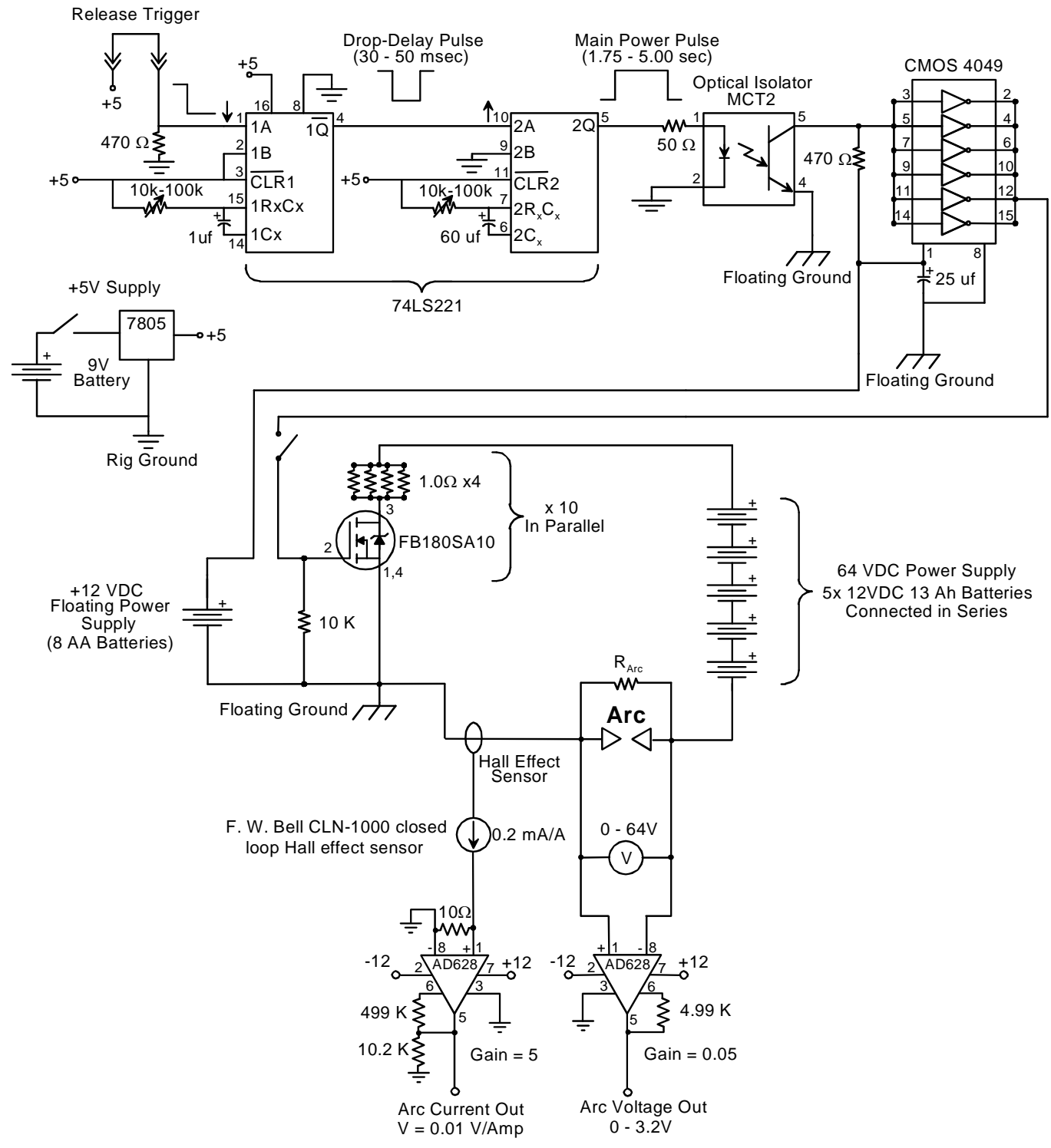

Figure 3 Electrical Schematic of the drop trigger, timing pulse, and high power switch used in the Zero Gravity Facility, NASA Glenn Research Center. 
a $10 \Omega$ shunt resistor allowing precision measurements of currents up to $500 \mathrm{~A}$. Initial testing with the outputs shorted confirmed that about $800 \mathrm{~A}$ could be switched. Because of the large charge stored in the batteries, the current and voltage during the 5-second pulse remained nearly constant. A series of current measurements into a constant load using different battery voltages allowed the internal resistance of the battery pack to be determined as a function of its charge state. Using mini-electrodes with $4.7 \mathrm{~mm}$ anodes, reproducible arc discharges with currents between 200 and 300 A could then be obtained by carefully adjusting the battery charging voltage.

When necessary, the battery pack was charged using $15 \mathrm{amp}$ adjustable autotransformer feeding a modular bridge rectifier to supply DC power to the batteries. The charging voltage was monitored with a digital voltmeter and limited to $65 \mathrm{~V}$ by a mechanical stop added to the Variac knob.

\section{E. Drop rig apparatus and instrumentation}

The same drop rig was utilized for both the 2.2 and 5 -second drop towers, and the capacitor and battery powered rigs had only minor differences. The capacitor bank was replaced by the battery pack, which although much heavier, had nearly the same dimensions and is shown in Figure 4. Changes to the power switch described previously were internal to the switch chassis. The only other difference was substitution of a slightly bigger vacuum chamber, from 2.8 to 7.5 liters, to allow more room for the expanding reaction zone produced by the larger arc. A schematic diagram of the larger arc apparatus is shown in Figure 1. Argon and vacuum for purging and running the arc were supplied on the ground, and the chamber was sealed before loading apparatus into the drop tower. The high power discharge created a rapid pressure rise in the chamber, which was monitored with an 0-50 PSIA strain gauge pressure transducer. The 4-20 mA output was converted to 0-5 V using a precision current loop receiver. The safe operating range of the chamber was vacuum to 65 PSI differential, which was ensured by an overpressure relief valve. The pressure spike generated by the arc could be partially controlled by venting through a fast acting 0-30 psig backpressure regulator. To prevent soot from discharging, the exhaust was filtered with a high conductance oil mist eliminator. Vented gas was directed through a tee to prevent any net force from affecting the flight of the drop rig. In practice, the regulator required about $1 \mathrm{sec}$ to begin regulating, and a pressure rise was still observed due to the finite response time and conductance of the filter and piping.

Observation of the arc was provided by two $3 / 4$ " thick Plexiglas windows, and standard interlaced video was recorded at 30 frames/sec using both black and white and color video cameras that where interchanged to look at the arc from either the side or end on. The end view had the advantage of blocking the direct view of the arc discharge and allowed a better view of the expanding plasma, while the side view allowed the evaporation of the anode to be observed. Because of the short observation time, video is hampered by the camera's automatic gain control circuit and iris, which must go from complete darkness to viewing the intense arc in a fraction of a second. In this regard, the B/W camera had a much quicker response time and dynamic range. Observation of the 5 -second arc was further hindered by the large amount of soot produced, which blocked the arc before the run was completed. The color camera allowed some differentiation of temperature to be discerned across the reaction zone, which appeared blue at the middle and transitioned to orange at the edge. Video was transmitted to the recorders by optical fibers in the 2.2-second drop tower and by infrared transmitters in the 5-second drop tower. The video was de-interlaced to 60 frames/sec using standard interpolation methods. High-speed video of the arc at $1000 \mathrm{frames} / \mathrm{sec}$ was obtained for several 2.2-second drops using a high-speed camera, which stored the video data in internal memory for downloading after the drop. Arc current, voltage, and chamber pressure was recorded for 5-second drops using a 100 sample/sec 16 bit ADC controlled by an onboard microprocessor that saved the data in internal memory for retrieval after the drop rig was recovered from the drop tower vacuum chamber. 


\section{Microgravity Nanotube Generator Rig (front view)}

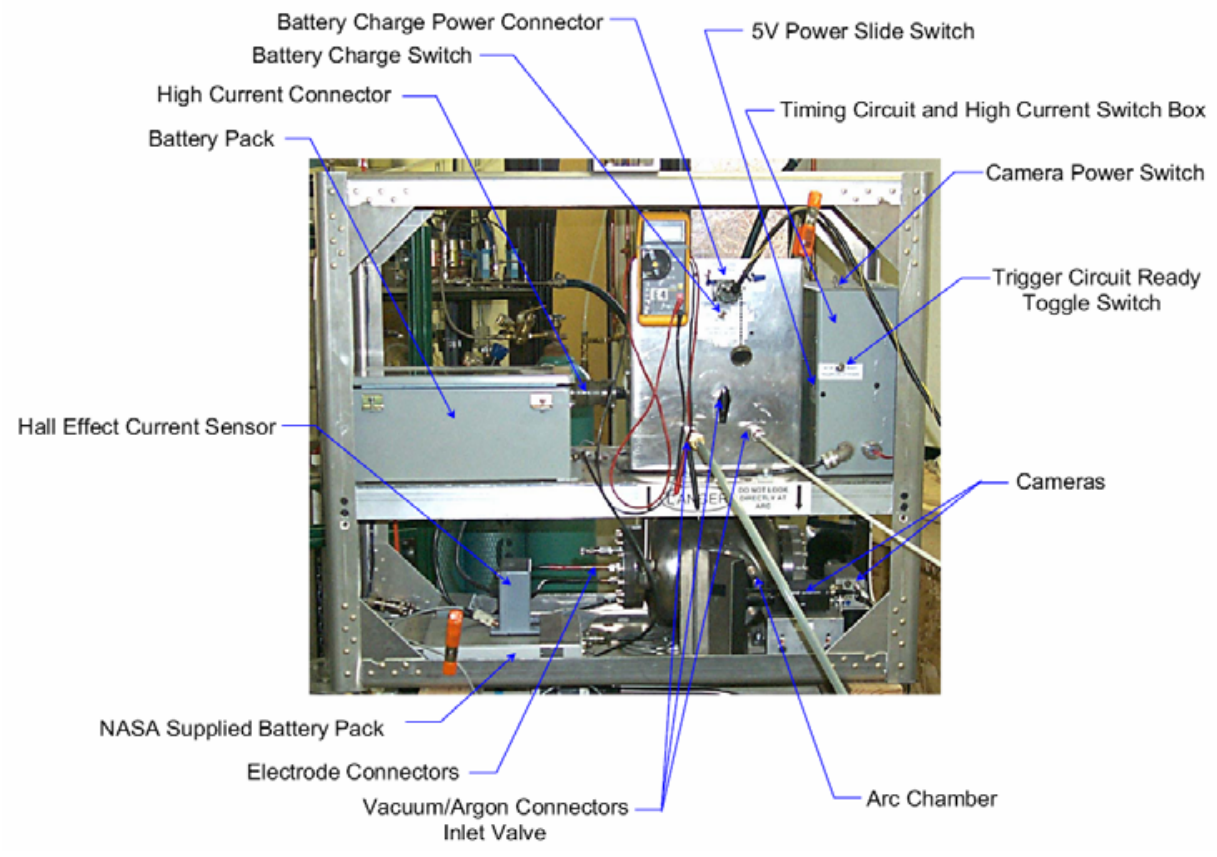

\section{Microgravity Nanotube Generator Rig (back view)}

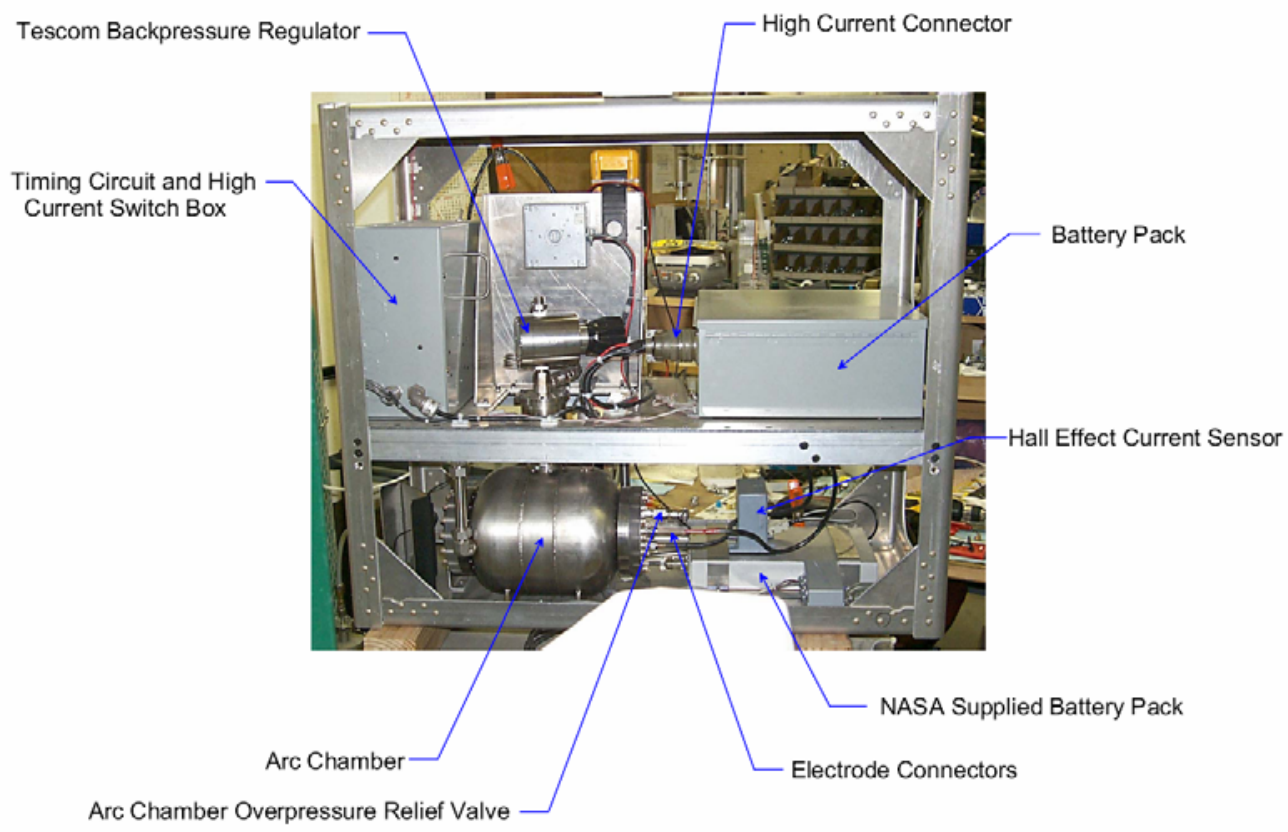

Figure 4 Photographs of the front and back views of the Microgravity Nanotube Drop Tower Rig. 


\section{EXPERIMENTAL RESULTS AND DISCUSSION}

Under normal gravity conditions, a SWNT forming carbon arc discharge looks very much like a sooting diffusion flame ${ }^{38}$ as shown by the first still frame in Figure 5 taken from a video of a 2.1 second discharge from the capacitively powered mini arc in normal gravity. Measurements confirm the rapid development of convective flow with a velocity of several meters per second above the discharge. A frame from the 2.2-second microgravity arc using identical discharge parameters is shown in the second part of Figure 5 and illustrates the dramatic difference the lack of convection makes to the hot gases. As expected, the plasma and reaction zone becomes a nearly spherical ball with large amounts of soot condensing at the edges. However, examination of the soot from both arcs using a HR-TEM with a resolution of $1 \mathrm{~nm}$ revealed nearly identical compositions made up of spherical soot particles 50-100 $\mathrm{nm}$ in diameter, Figure 7a. Each particle appeared to be composed of highly amorphous carbon structures and contained one or more $5-10 \mathrm{~nm}$ diameter catalyst particles. Examination of the catalyst particles using X-ray fluorescence from the electron beam revealed both $\mathrm{Ni}$ and $\mathrm{Y}$, as expected. No nanotubes were observed in either the normal or microgravity 2.1 second samples. It is believed that even with precautions to limit heat losses, the small amount of power from the capacitively powered mini arc caused very rapid condensation of the carbon and prevented nanotube growth. This is consistent with the large amount of amorphous carbon surrounding the catalyst particles, suggesting that the metal catalyst solidified before nanotube growth started.

Given this result, the arc was enlarged, the power was increased by about 15 times, and the run was lengthened to 5 -seconds as described previously to increase the temperature in the reaction zone. The discharge parameters from a typical 5 -second arc ( $4.85 \mathrm{sec}$ in the drop tower) powered by the battery pack charged to $60 \mathrm{~V}$ is shown in Figure 6. The current and voltage, $225 \mathrm{~A}$ at $26 \mathrm{~V}$, was adjusted to yield smooth evaporation of the anode for the entire discharge time. For investigating the time evolution of the temperature, pressure, and reactants in the chamber, it was experimentally desirable to keep the power input into the chamber constant, rather than keeping the current constant. As shown in Figure 6, the arc power of nearly $6 \mathrm{~kW}$ is surprisingly constant. This is actually an important benefit of this type of simple, unregulated power supply. As can be seen, the operating voltage of $26 \mathrm{~V}$ is very nearly one half of the open circuit battery voltage. With the arc resistance forming one half of a voltage divider composed of the arc, battery, switch, wiring, and connection resistances, it can be shown mathematically that the power change for small changes in the arc resistance is zero when the arc resistance equals the remaining circuit resistance. In this run, the arc resistance comprised $43 \%$ of the total $0.267 \Omega$ circuit resistance, and the rate of power change with change in the arc resistance was about 7.5 times smaller than what would be obtained with a constant current source. In practice, as the discharge gap narrows, the resistance increases, causing the voltage to increase and current to decrease, while the opposite happens when the gap increases, yielding nearly constant power dissipation for small variations of the contact arc. The large spikes in the current and voltage are thought to be points where the slide momentarily sticks while the continuous oscillations are due to mechanical vibrations of the spring and holder. It was experimentally observed that higher spring constants, which forced the electrodes together more tightly, raised the voltage, dropped the current, and increased the carbon vaporization rate. Up to one gram of carbon could be vaporized in 5-seconds using a stiff spring. However, a contact arc failed to form during many of the runs using stiff springs, resulting in only resistive heating with no carbon vaporization. The above conditions using a looser spring provided very reproducible runs in the 5-second drop tower that vaporized about $2 \mathrm{~mm}$ or 60 mg of anode rod per second.

HR-TEM analysis of the soot collected from the chamber of five second arc runs in both normal and microgravity showed small quantities SWNTs occurring in bundles of two or three tubes, Figure $7 \mathrm{~b}$. No significant difference was discerned between the normal and microgravity environments. The soot also contained a large amount of amorphous carbon coated catalyst particles similar to those observed in the 2.2-second drop tower using the low power apparatus. In addition, it also contained a significant number of "sea urchin" like particles in which multiple SWNTs started to grow from the catalyst particles, but were then quenched, Figure 7c and 7d. These observations are again consistent with an environment in which small carbon clusters begin condensing on the liquid $\mathrm{Ni} / \mathrm{Y}$ catalyst particles, and SWNTs start to grow, but then the reaction is quenched before significant growth can occur. This indicates that although buoyancy induced convection has been eliminated, the gas near the arc still cools too fast for significant nanotube growth to occur. This rapid cooling can be ascribed to two main factors: transient heating effects which prevent the gases from attaining a high enough temperature for sustained nanotube growth in the short microgravity time frame and jets of vaporized carbon that are ejected into the cold buffer gas from the vaporizing anode. 


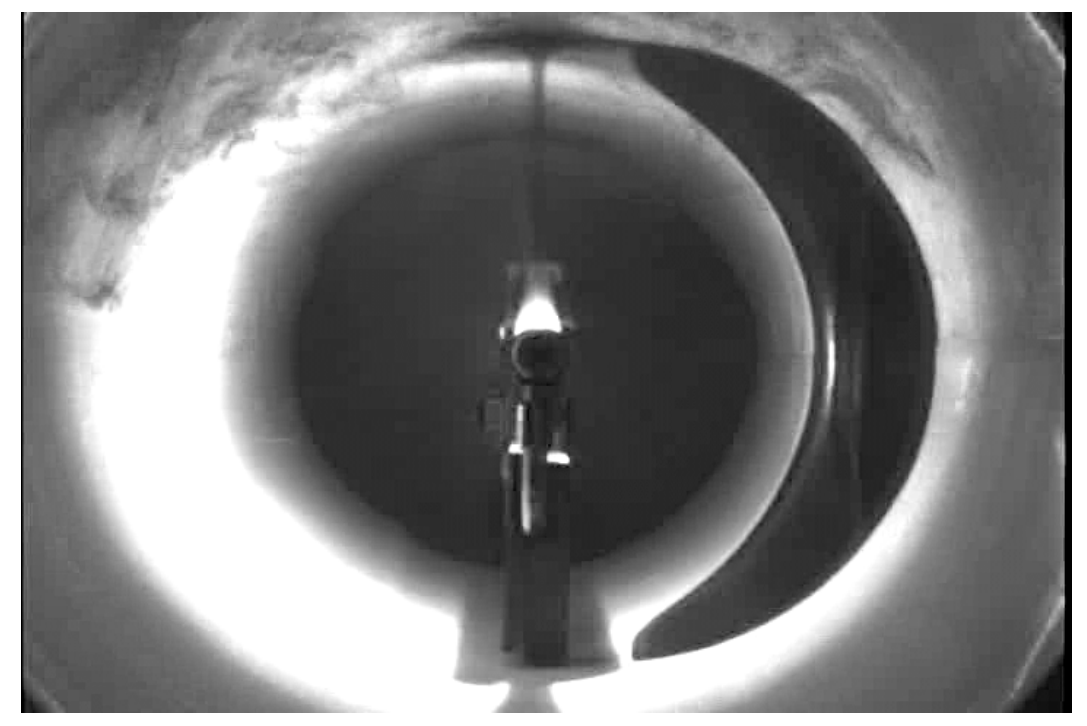

$1 \mathrm{~g}$

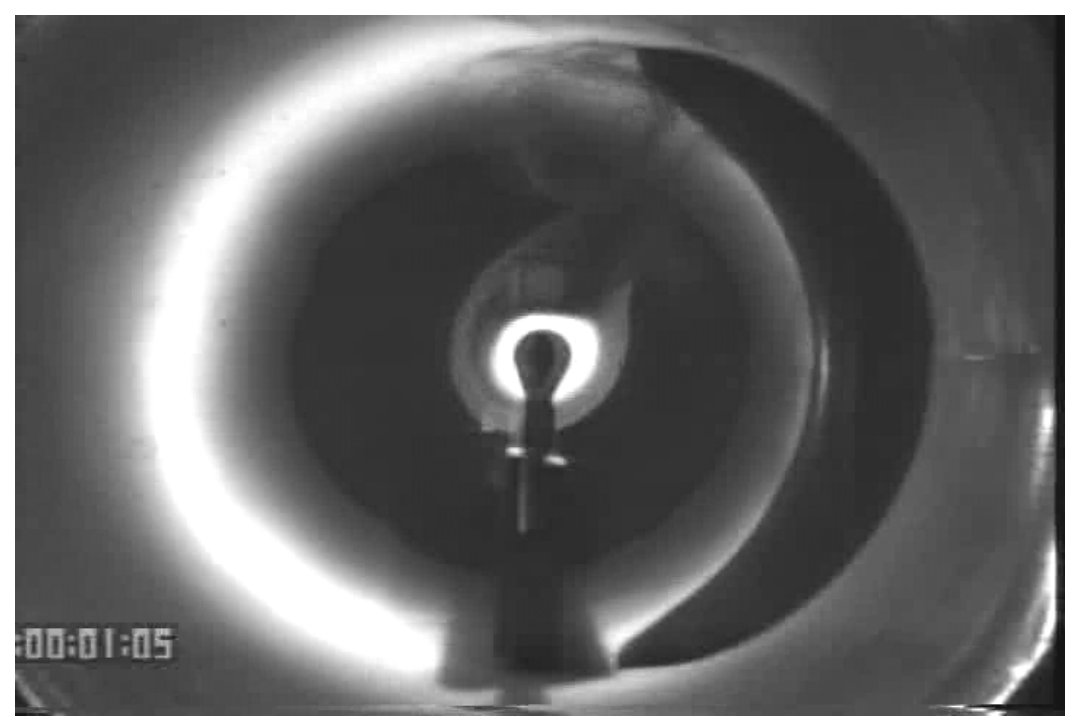

microgravity

Figure 5 Video stills of 2.2-second arc discharges illustrating the difference between the $1 \mathrm{G}$ arc and the microgravity arc.

The detrimental effect of the heating transient on the drop tower arc can be deduced by closely examining previous arc experiments. The single prior study of a reduced gravity arc in the oscillating reactor employed approximately $20-40$ amps of current for 1.0 second of the 2.3 second oscillation period. ${ }^{26}$ For a 30 minute run, the integrated reduced gravity arc time was 13 minutes. Power delivery into the apparatus was not reported, but a typical value assuming a $25 \mathrm{~V}$ drop across the arc would indicate up to 800 watts and $800 \mathrm{~J}$ per oscillation cycle would be deposited into the arc chamber. With a 13 minute integrated arc time, this amounts to over $600 \mathrm{~kJ}$ total energy deposited into the chamber, which had a volume of only 1.6 liters. Temperature and pressure data of the buffer gas as a function of the run time was not provided, but the inert gas in the chamber could easily have reached between $500-1000^{\circ} \mathrm{C}$, substantially lessoning the temperature gradient. The wall temperature of the reactor was also not reported, but could be substantial considering cooling was by natural convection along the outer wall with insulating 
soot covering the inner wall, helping to further reduce heat losses. A similar situation applies to our conventional apparatus run under normal gravity, which had $3 \mathrm{~kW}$ of power applied for 2 minutes, depositing $360 \mathrm{~kJ}$ of energy into the chamber. In each case, a large amount of time was provided for the buffer gas to attain fairly high temperatures, dramatically increasing the size of the heated zone and reaction time for nanotube growth. The drop tower microgravity arc experiment, by contrast, is extremely short, and the buffer gas into which the carbon vapor expands is essentially at room temperature, producing a very large temperature gradient.

With the removal of the large convective flow by the microgravity environment, large jets of carbon vapor could now be readily observed emanating from the evaporating anode in both the normal and high-speed video of the
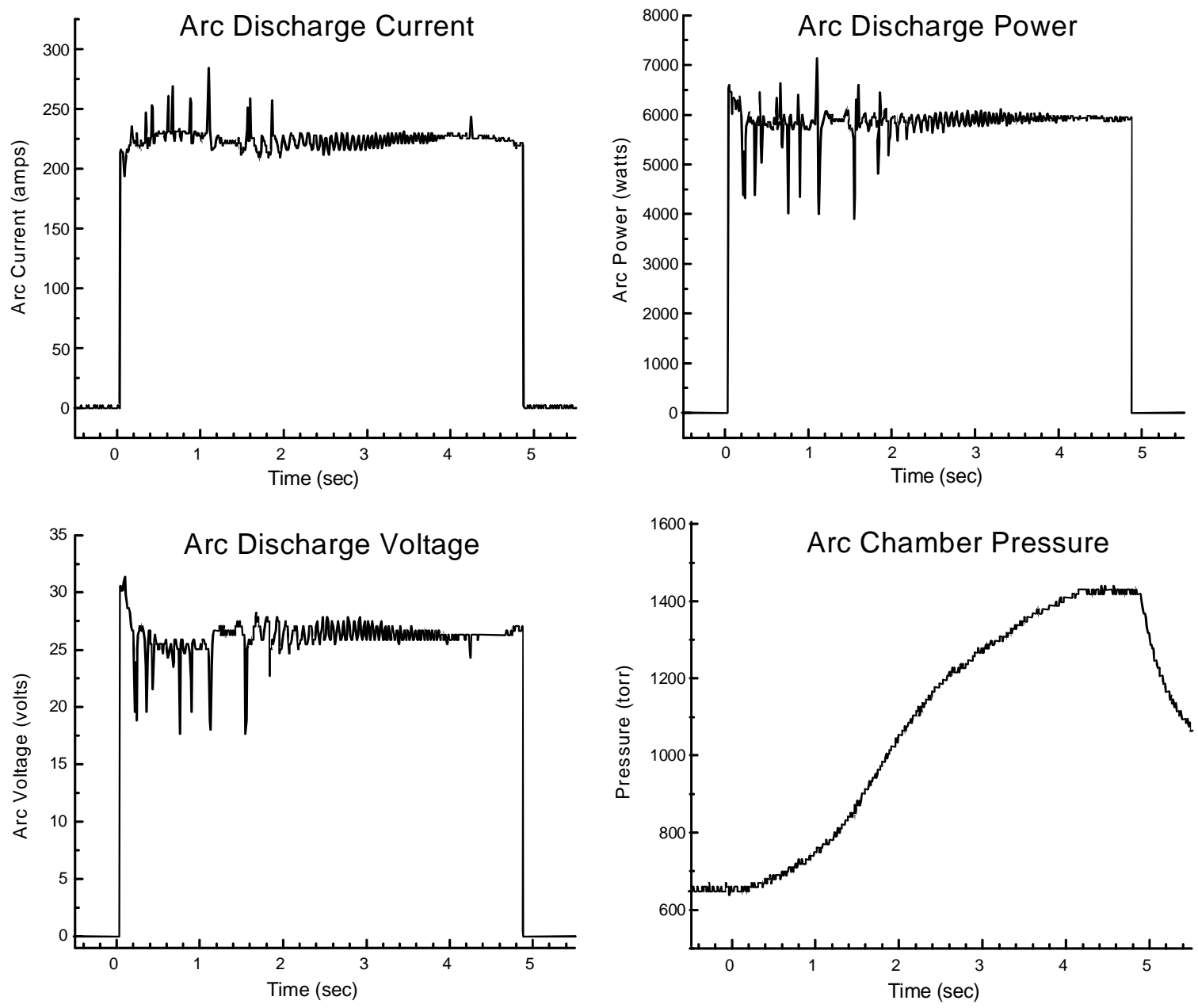

Figure 6 Key electrical parameters for the microgravity miniature carbon arc.

microgravity arcs. These vapor jets could extend several centimeters or more from the arc, and while not as dramatic as the convective flow in normal gravity, they still formed vortices that mixed the carbon vapor with the background gas, causing rapid cooling. While the arc appears steady to the naked eye, high-speed video shows that it jumps around on the short time scale. As a particular location on the anode is evaporated, it exhibits a lower electric potential, and the arc soon jumps to a higher spot with a greater electric field. While not considered in the previous analysis of the convective flow in the arc discharge, ${ }^{21}$ diffusion of evaporated reactants from the anode is substantial in this case. Evaporation of $60 \mathrm{mg} / \mathrm{sec}$ of carbon creates over one liter/sec of carbon vapor that must move away from the arc before cooling and condensing as soot and SWNTs. The small gap of the contact arc exacerbates the problem and results in confined jets of hot vapor randomly flowing in alternate directions from the anode for 10's to 100's of milliseconds before changing direction as the anode evaporated. These initial jets can be seen mixing with the buffer gas and condensing as soot along the boundary. The time average of these jets 
A

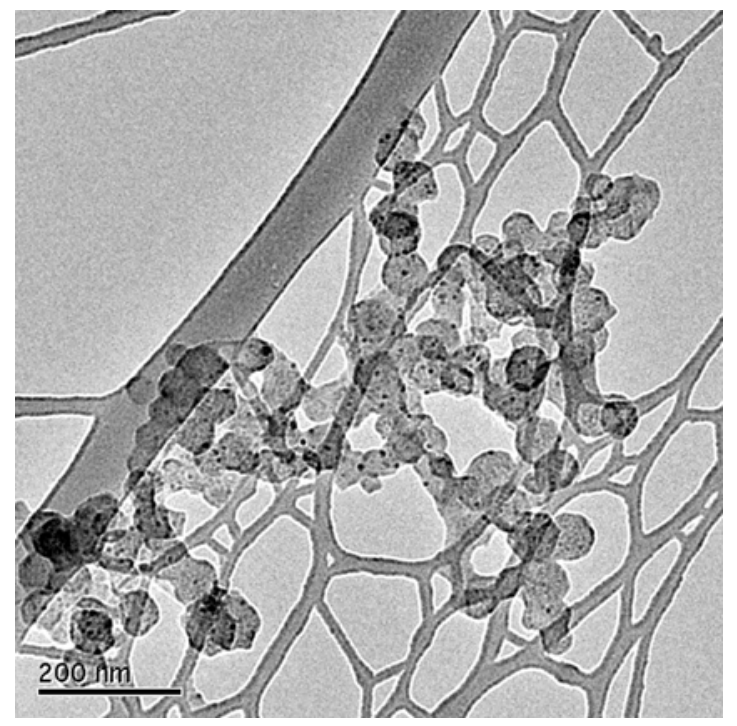

C

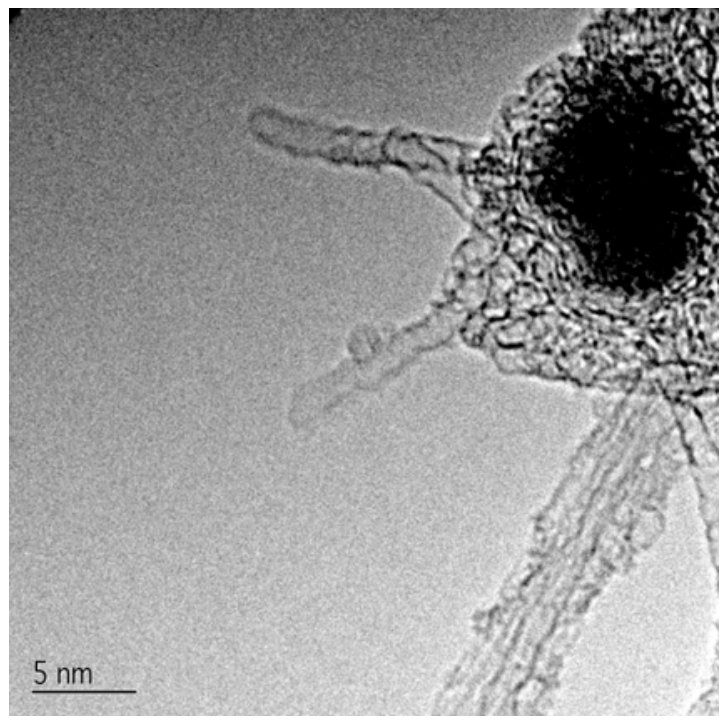

B

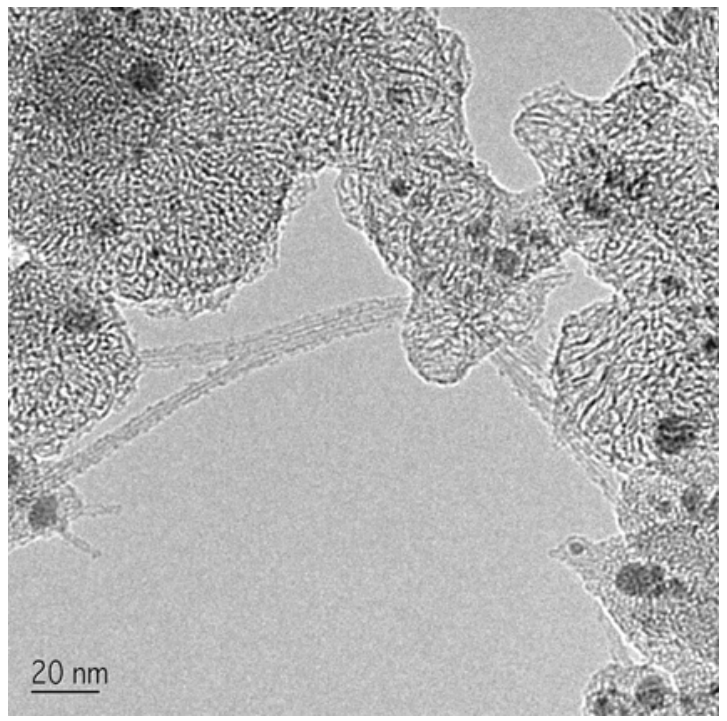

$\mathrm{D}$

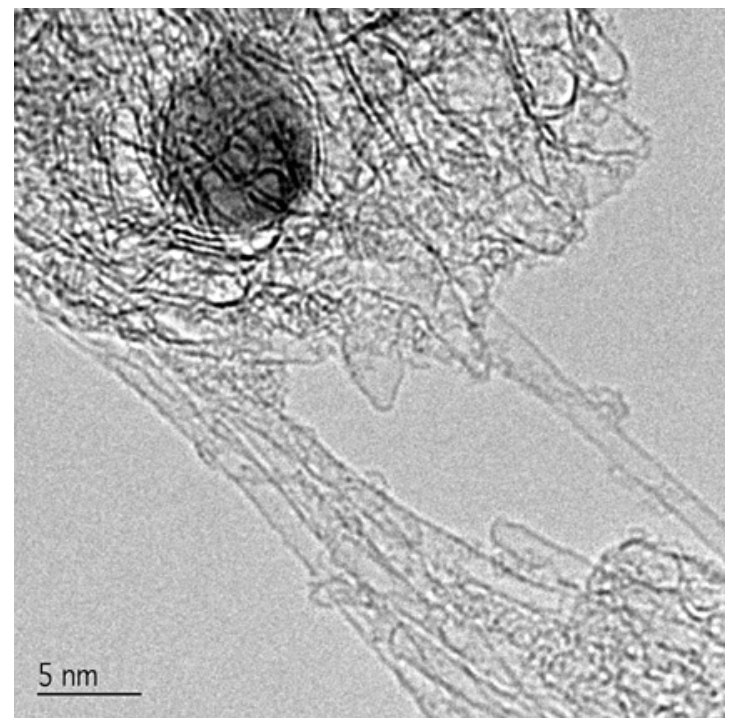

Figure 7 A) Primary soot particles from a typical 2.1-second run. The branching structures are the holey carbon support. B) Primary soot particles from a 4.85 second microgravity run showing SWNTs. C) \& D) Sea urchin type SWNT particles and short nanotubes. 
eventually produces a spherically shaped reaction zone around the arc, but the formation took several seconds or longer.

Both of these observations indicate that nanotube growth is critically dependent on maintaining the proper growth temperature, agreeing with observations of rate limiting processes for nanotube growth performed in the laser oven apparatus. ${ }^{39}$ At too high a temperature, the carbon dissolved in the metal catalyst is not supersaturated, reducing the driving force for forming the nanotube. At too low a temperature, the catalyst particle solidifies, quenching the nanotube growth. The optimum temperature depends on the catalyst mixture, buffer gas type and pressure, and is not precisely known. A good estimate is around $1200^{\circ} \mathrm{C} .{ }^{39}$ In principle, by eliminating thermal convection, microgravity should dramatically help stabilize the temperature and concentration gradient around the arc. However, the transient arcs studied in these experiments did not have enough time to establish the necessary high temperature and gradual temperature gradient needed for extended nanotube growth. The microgravity environment may indeed allow very high nanotube growth times under nearly ideal conditions, but it will require much longer times than the 5 -seconds available in the drop tower. Ideally, evaporation of the electrodes will need to be performed in a buffer gas maintained at the optimal $1200^{\circ} \mathrm{C}$ growth temperature. Microgravity conditions amenable to SWNT growth might be generated on the zero-G aircraft, but more likely would need to be performed on the international space station or space shuttle.

\section{ACKNOWLEDGMENTS}

The authors thank the technicians at the NASA Glenn 2.2-second and 5-second drop towers for providing invaluable assistance with the apparatus and use of the drop tower facilities. Dave Hull, NASA GRC, is given special appreciation for performing the HR-TEM analysis. This work was supported by NASA grant NAS3-99142 with Dr. Douglas Feikema as Technical Monitor.

\section{REFERENCES}

${ }^{1}$ S. Iijima, "Helical Microtubules of Graphitic Carbon," Nature 354, 56 (1991).

${ }^{2}$ S. Iijima and T. Ichihashi, "Single-shell carbon nanotubes of 1-nm diameter," Nature 363, 603 (1993).

${ }^{3}$ D. S. Bethune, C. H. Kiang, M. S. de Vries, G. Gorman, R. Savoy, J. Vazquez, and R. Beyers, "Cobalt-catalysed growth of carbon nanotubes with single-atomic-layer walls," Nature, 363, 605 (1993).

${ }^{4}$ P. Ajayan, "Nanotubes from Carbon," Chem. Rev. 99, 1787 (1999).

${ }^{5}$ T.W. Ebbeson (Ed.) Carbon Nanotubes, their Preparation and Properties, CRC Press, Boca Raton, FL, 1997.

${ }^{6}$ R. Satio, M. Dresselhaus, G. Dresselhaus Physical Properties of Carbon Nanotubes, Imperial College Press, London, 1998.

${ }^{7}$ S. Kumar, T. D. Dang, F. E. Arnold, A. R. Bhattacharyya, B. G. Min, X. Zhang, R. A. Vaia, C. Park, W. W. Adams, R. H. Hauge, R. E. Smalley, S. Ramesh, and P. A. Willis, "Synthesis, structure, and properties of PBO/SWNT composites", Macromolecules, 35, 9039 (2002).

${ }^{8}$ L. M. Ericson, H. Fan, H. .Peng, V. A. Davis, W. Zhou, J. Sulpizio, Y. Wang, R. Booker, J. Vavro, C. Guthy, A. N. G. Parra-Vasquez, M. J. Kim, S. Ramesh, R. K. Saini, C. Kittrell, G. Lavin, H. Schmidt, W. W. Adams, W. E. Billups, M. Pasquali, W.-F. Hwang, R. H. Hauge, J. E. Fischer and R. E. Smalley, "Macroscopic, neat, singlewalled carbon nanotube fibers," Science, 305, 1447 (2004).

${ }^{9}$ J. Hone, M. Whitney, C. Piskoti and A. Zettl, "Thermal conductivity of single-walled carbon nanotubes," Phys. Rev. B, 59, R2514 (1999).

${ }^{10}$ W. Liang, M. Bockrath, D. Bozovic, J. H. Hafner, M. Tinkham and H. Park, "Fabry-Perot interference in a nanotube electron waveguide," Nature 411, 665 (2001).

${ }^{11}$ S. J. Tans, A. R. M. Verschueren and C. Dekker, "Room-temperature transistor based on a single carbon nanotube," Nature 393, 49 (1998).

${ }^{12}$ A. Bachtold, P. Hadley, T. Nakanishi, and C. Dekker, "Logic circuits with carbon nanotube transistors," Science 294, 1317 (2001).

${ }^{13}$ B. M. Segal, D. K. Brock and T. Rueckes, "Hybrid circuit having nanotube electromechanical memory," US Patent 6,836,424, Nantero, Inc., Woburn, MA, (2004).

${ }^{14}$ Noritake Corp., Carbon Nanotube Field Emission Lamp, Noritake Corporation, Nagoya, Japan, cnt@noritakeitron.jp.

${ }^{15}$ C. Journet, W. K. Maser, P. Bernier, A. Loiseau, M. Lamy de la Chapelle, S. Lefrants, P. Deniard, R. Lee, and J. E. Fischer, "Large-scale production of single-walled carbon nanotubes by the electric-arc technique," Nature 388, 756 (1997). 
${ }^{16}$ T. Guo, P. Nikoleav, A. Thess, D. T. Colbert, and R. E. Smalley, "Catalytic growth of single-wall carbon nanotubes by laser vaporization," Chem. Phys. Lett. 243, 49 (1995).

${ }^{17}$ J. F. Colomer, C. Stephan, S. Lefrant, G. Van Tendeloo, I. Willems, Z. Konya, A. Fonseca, Ch. Laurent, and J.B. Nagy, "Large-scale synthesis of single-wall carbon nanotubes by catalytic chemical vapor deposition (CCVD) method," Chem. Phys. Lett. 317, 83 (2000).

${ }^{18} \mathrm{P}$. Nikolaev, "Gas-phase production of single-walled carbon nanotubes from carbon monoxide: a review of the HiPco process," J. Nanosci. Nanotechnol. 4, 307 (2004).

${ }^{19}$ M. D. Diener, N. Nichelson, and J. M. Alford, "Synthesis of Single-Walled Carbon Nanotubes in Flames," J. Phys. Chem. B 104, 9615 (2000).

${ }^{20}$ T. M. Gruenberger, J. Gonzalez-Aguilar, F. Fabry, L. Fulcheri, E. Grivei, N. Probst, G. Flamant, H. Okuno and J.C. Charlier, "Production of carbon nanotubes and other nanostructures via continuous 3-phase AC plasma processing," Fullerenes, Nanotubes, and Nanostructures, 12, 571 (2004).

${ }^{21}$ S. Usuba, H. Yokoi and Y. Kakudate, "Numerical analysis on the dispersion process of carbon clusters synthesized by gas evaporation using DC arc", J. Appl. Phys., 91, 12, 10051, (2002).

${ }^{22}$ A. A. Puretzkey, D. B. Geohegan, X. Fan, and S. J. Pennycook, "Dynamics of single-wall carbon nanotube synthesis by laser vaporization," Appl. Phys A. 70, 153 (2000).

${ }^{23} \mathrm{~S}$. Aoyama and T. Mieno, "Effects of gravity and magnetic field in production of $\mathrm{C}_{60}$ by a DC arc discharge", Jpn. J. Appl. Phys., 38, 267 (1999).

${ }^{24}$ C. Marin, M. D. Serrano, N. Yao and A. G. Ostrogorsky, "Convection-assisted synthesis of small-diameter singlewalled carbon nanotubes by the electric arc technique, in the vertical configuration", Nanotechnology, 13, 218 (2002).

${ }^{25}$ T. Mieno, N. Matsumoto and M. Takeguchi, "Efficient production of single-walled carbon nanotubes by J x B gasarc discharge method", Jpn. J. Appl. Phys., 43, 1527 (2004).

${ }^{26} \mathrm{~T}$. Mieno, "Characteristics of the gravity-free gas-arc discharge and its application to fullerene production", Plasma Phys. Control. Fusion, 46, 211 (2004).

${ }^{27} \mathrm{~T}$. Mieno, "Efficient production of endohedral metallofullerenes in repetitive gravitation-free arc discharge using a vertical swing tower," Jpn. J. Appl. Phys., 37, 761 (1998).

${ }^{28}$ M. Kanai, A. Koshio, H. Shinohara, T. Mieno, A. Kasuya, Y. Ando, and X. Zhao, "High-yield synthesis of singlewalled carbon nanotubes by gravity-free arc discharge", Appl. Phys. Lett., 79, 18, 2967 (2001).

${ }^{29}$ T. Mieno, "Gas Temperature evolution of the gravity-free gas arc discharge under a parabolic flight of jet plane", Jpn. J. Appl. Phys., 42, 960 (2003).

${ }^{30}$ S. Benyoub, J.-M. Beuken, J.-C. Charlier, J.-P. Minet and J.-P. Issi, "Growth and agglomeration of carbon materials under microgravity conditions," Lab. of Physical Chemistry and Physics, University of Louvain-Neuve, 1, Place Croix du Sud Louvain-la-Neuve, Belgium.

${ }^{31}$ J. Lekan, D. J. Gotti, A. J. Jenkins, J. C. Owens and M. R. Johnston, "Users Guide for the 2.2- second drop tower of the NASA Lewis Research Center," NASA Technical Memorandum 107090 (1996).

${ }^{32}$ D. M. Thompson, “Zero gravity research facility user's guide," NASA Technical Memorandum 1999-209641, (1999).

${ }^{33}$ POCO Graphite Inc., AXF-5Q grade graphite, 300 Old Greenwood Road, Decatur, TX 76234.

${ }^{34}$ HSE Chemistry 5, Outokumpu Research Oy, Pori, Finland (2002).

${ }^{35}$ A. S. Koch, K. C. Khemani, and F. Wudl, "Preparation of Fullerenes with a Simple Benchtop Reactor," J. Org. Chem. 56, 4543 (1991).

${ }^{36}$ A. V. Krestinin, M. B. Kislov and A. G. Ryabenko, "On the mechanism of single-walled carbon nanotube nucleation in the arc and laser processes: Why bimetallic catalysts have high efficiency," Nanoengineered nanofibrous materials, Kluwer Academic Book Publishers, Dordrecht, Netherlands, 169, 107 (2004).

${ }^{37}$ W.D. Cagle, T.P. Thrash, J.M. Alford, L.P.F. Chibante, G.J. Ehrhardt, and L.J. Wilson (1996). "Synthesis, Characterization, and Neutron Activation of Holmium Fullerenes", J. Am. Chem. Soc. 118, 8043.

${ }^{38}$ Sunderland, P. B., R. L. Axelbaum, D. L. Urban, B. H. Chao and S. Liu, "Effects of structure and hydrodynamics on the sooting behavior of spherical microgravity diffusion flames," Combustion and Flame, 132, 25 (2003).

${ }^{39}$ Jost, O., A. A. Gorbunov, J. Möller, W. Pompe, X. Liu, P. Georgi, L. Dunsch, M. S. Golden, and J. Fink, "Ratelimiting processes in the formation of single-walled carbon nanotubes: Pointing the way to the nanotube formation mechanism," J. Phys. Chem. B 106, 2875 (2002). 